\title{
Temporal integration of monocular images separated in time: Stereopsis, stereoacuity, and binocular luster
}

\author{
IRA LUDWIG \\ Philipps-Universität Marburg, Marburg, Germany \\ Wolfgang Pieper \\ Justus-Liebig-Universität Geissen, Giessen, Germany \\ AND \\ HARALd LACHNIT \\ Philipps-Universität Marburg, Marburg, Germany
}

\begin{abstract}
We evaluated stereopsis and binocular luster using electronically controlled shutter glasses with alternating monocular stimulation. In Experiment 1, we used the standard method for testing stereoacuity to obtain a gradual measure of stereopsis. Stereo thresholds decreased with increasing alternating frequency of two monocular half-images without a delay between them. Increasing delays led to increasing thresholds. In Experiment 2 , we compared stereopsis resulting from two monocular half-images of a random-dot stereogram and binocular luster with respect to the minimum alternating frequency of the two half-images and the maximum interocular delay that were tolerated without a breakdown of the impression. Below $3 \mathrm{~Hz}$, no stereopsis occurred. Binocular luster was observed only above $10 \mathrm{~Hz}$. The mean threshold of interocular delay for detecting the global figure in a random-dot stereogram was about $51 \mathrm{msec}$, but for binocular luster it was about $20 \mathrm{msec}$. Overall, temporal integration was better for stereopsis than for binocular luster.
\end{abstract}

Stereopsis, or stereo vision, is a result of the fact that the two eyes view visual stimuli from slightly different positions and, therefore, the two retinal images are disparate. Obviously, our visual system compares the two monocular images and extracts binocular disparities from them in order to generate stereo vision. But what would happen if the two monocular views were not present simultaneously but staggered in time? Would this hinder the extraction of binocular disparity? As an example, imagine looking through a fence close to your eyes, with picket and gap widths identical to the interpupillary distance. With respect to the scene behind the fence, your vision would be monocular, since when one eye was looking at the scene through a gap the other eye's view would be blocked by a picket. If you moved parallel to the fence, you would get a sequence of several pictures of the scene, but all of them would be monocular. Would you still have a stereoscopic impression of the scenery behind the fence, and, if so, would this depend on a certain speed of movement? More generally, would stereovision occur when the two monocular images were separated in time, and, if so, would it be tied to a certain presentation time of each monocular image or to a certain frequency of alternation between the two images?
The answer to the first question seems to be "yes," since a corresponding phenomenon was qualitatively described more than a century ago (Dvorák, 1872; Ewald \& Gross, 1906; Exner, 1875; Guilloz, 1904; Stevenson \& Sanford, 1908). More recent studies offer quantitative analyses of stereopsis from alternating monocular stimulation by considering its dependence on, for example, the presentation duration of the two monocular images (corresponding to the frequency of alternation between them) and the duration of delays between them. They focus either on the occurrence of stereopsis per se (Aulhorn, 1971; Efron, 1957; Engel, 1970; Herzau, 1976; Ross \& Hogben, 1974) or on the measurement of stereoacuity (Ogle, 1963; Wist \& Gogel, 1966). As we will elaborate later on, in the present article we continue the quantitative analysis of temporal integration of monocular images by (1) asking whether stereopsis undergoes an abrupt breakdown or a gradual deterioration in certain stimulation conditions and (2) extending the range of binocular phenomena studied under conditions of alternating monocular stimulation.

Let us start with a sketch of studies focusing on stereopsis per se. Efron (1957) used a rotating disk to display monocular images alternately to each eye. When a single pair of monocular images was used, stereoscopic fusion 
occurred with maximum delays of $6-13 \mathrm{msec}$. With a sequence of pairs, Efron found a reciprocal relation of the within-pair and between-pairs intervals. Aulhorn (1971) reported that stereoscopic vision is possible with monocular alternating presentation at frequencies as low as 4-5 Hz. Herzau (1976) found that without an interocular delay, stereopsis was evident up to maximum durations of the monocular images of about $370-480 \mathrm{msec}$. With shorter stimulus durations, increasingly longer interocular delays were tolerated without loss of stereopsis. Undisturbed quantitative depth localization required a frequency of about $2 \mathrm{~Hz}$ higher than that for the mere occurrence of stereopsis. In all three studies just described, disparate stimuli containing monocular visible contours were used. In contrast, Engel (1970) as well as Ross and Hogben (1974) used random-dot stereograms, introduced by Julesz (1960), to dismiss monocular depth cues. Engel reported stereoscopic depth with stimulus repetition rates between 2.5 and $10 \mathrm{~Hz}$ measured at one eye. Ross and Hogben found the discrimination of a disparate target to be almost unaffected by interocular delays below the range of 36-72 $\mathrm{msec}$. Discrimination got increasingly worse with longer delays, and it was near chance level with delays of $150 \mathrm{msec}$.

Let us now consider studies in which stereoacuity was measured with alternating monocular stimulation. Ogle (1963) presented both the monocular images of a test line and a binocular visible comparison line to both eyes and asked his participants to judge which of the two lines was closer. Stereo thresholds increased with increasing interocular delay. The thresholds decreased with increasing exposure times of the monocular images, obviously due to the increasing temporal overlap between the two images. Wist and Gogel (1966) manipulated the delays within and between pairs of alternating monocular images of a luminous disc and measured observers' ability to place that disc (which emerged by fusion) at the apparent depth of a binocularly viewed comparison object. They found that stereoacuity was hardly affected by interocular delays up to $32 \mathrm{msec}$. Stereoacuity, however, was degraded with delays above this value and likewise decreased with increasing intervals between the successive image pairs.

Many of the studies described above have methodological shortcomings that might have influenced the results. The use of mechanical diaphragms prevents the frequency of the monocular images' presentation from being controlled independently of the interocular delay. In some studies in which rotating sectored disks were used, several variables were confounded as well. In Efron's (1957) study, changes in the rotation speed altered not only the interval within a pair of flashes but also that between consecutive pairs, the ratio of these two intervals, and the flash duration, whereas changes in flash duration simultaneously altered the two intervals and their ratio as well as the brightness of the field. Similar techniques, used by Stevenson and Sanford (1908) and by Herzau (1976), for example, confounded at least interocular delays and stimulus duration. Other mechanical devices, such as arrangements of rotating mirrors (Ogle, 1963), allow only a limited presentation frequency.
Therefore, we looked for another method that would allow precise and independent control of the stimulation conditions. In our opinion, the most promising devices for such purposes are electronically controlled liquid-crystal shutter glasses (see Mollon, Polden, \& Morgan, 1977), described by Milgram (1987). Such devices are able to independently open or close each of the two glasses in front of the eyes and thus selectively uncover or cover an observer's view of a target. Among other advantages, the device can rapidly switch between an open and a closed state with exact control of its activation. An open glass is transparent and thus hardly affects the perceived brightness, sharpness, and spectral composition of the viewed objects. A closed glass scatters incident light, resulting in a uniformly luminous texture and blocking all information in the observer's visual field. Such a device allows stereovision from alternating monocular images to be studied without confounding the relevant stimulation conditions.

A second methodological problem of the studies mentioned above concerns the way stereo vision was measured. In most of the studies, stereopsis was measured in an all-or-none fashion. In general, the authors of these studies stated that stereopsis was present with some durations of the monocular images (and with the respective frequencies of alternation between the two monocular images) and some delays between them, and not with others. But does this mean that there is an abrupt breakdown of stereopsis at a certain duration of the monocular images (or at a certain alternating frequency)? To answer this question, instead of considering stereopsis in an all-ornone fashion, one must consider the extent or precision of stereopsis - that is, stereothresholds or stereoacuity should be measured under varying durations of the monocular images. Only Ogle (1963) and Wist and Gogel (1966) did this, by measuring the extent of stereopsis. In addition to the methodological shortcomings mentioned above, however, in both these studies stereoacuity was determined in rather unusual ways: as dispersion in consecutive judgments (Ogle, 1963) and as dispersion in consecutive adjustments of the apparent depths of a test object and a comparison object (Wist \& Gogel, 1966).

Thus, the first aim of our study was to measure stereoacuity as the extent of stereopsis (instead of stereopsis in an all-or-none fashion) with a more standard method, such as that of the three-needle experiment introduced by Helmholtz (1910). Separation of the monocular stimuli through the use of electronically controlled shutter glasses should allow for measurement of stereoacuity with this standard method. Thus, we wanted to study stereoacuity in its dependence on the alternating frequency of monocular images (corresponding to the duration of the monocular images) and on interocular delay, since in the earlier studies mentioned above these two variables had been found to be the most influential ones. Due to considerable variation between observers, a larger number of observers than that used in Ogle's (1963) or Wist and Gogel's (1966) studies (1-4) should be used. In order to exclude monocular cues, it would be ideal to use a random-dot technique. However, gradually measuring stereoacuity is rather difficult 
with random-dot stereograms, since the pair of pictures constituting each random-dot stereogram always includes a fixed amount of disparity, which makes it impossible to vary disparity continuously, as we wanted to do with our method. We must make sure, however, that monocular cues will not affect our measurements.

The second aim of our study was to extend the range of phenomena studied under conditions of alternating monocular stimulation. The above-mentioned studies focused on stereopsis resulting from the binocular disparity between two monocular images. Interestingly, we found no information about what happens under such conditions to other binocular phenomena (e.g., binocular luster), especially when the monocular images do not entail binocular disparity. Such information, however, would be very important for clarifying the underlying processes. If the stereoscopic impression is due to a rather general process through which information about the first stimulus is stored over a limited time interval and integrated with information about the second stimulus (or directly with its image), then other binocular phenomena should occur under such conditions as well. If, however, specific processes tied to binocular disparity are responsible, other binocular phenomena should hardly be observable with alternating monocular stimulation. In addition, a comparison of the temporal characteristics of stereopsis and binocular luster could give us some more information about the underlying processes, since different temporal characteristics could indicate different processes underlying the temporal integration in these two phenomena.

\section{EXPERIMENT 1}

In Experiment 1, we evaluated stereovision by presenting alternating monocular half-images by means of electronically controlled shutter glasses. Instead of testing the mere occurrence of stereopsis, we preferred to obtain stereoacuity as a gradual measure of stereopsis under several conditions of alternating monocular stimulation using the standard method for testing stereoacuity. Following Helmholtz's (1910) three-needle set-up, we designed the three-rod apparatus described below. A strength of this method is that a real 3-D display is used instead of a CRT. In one condition, we varied the alternating frequency of two half-images without delay between them, whereas in another condition the interocular delay was manipulated.

\section{Method}

Participants. Fifteen students of psychology and employees of the University of Giessen ( 5 men and 10 women) took part in Experiment 1 . Their mean age was 26 years (range, 20-60 years). The students received course credit for their participation. All the participants were tested for normal visual acuity (left eye, right eye, and binocularly) and normal stereoscopic acuity (at least $19 \mathrm{sec}$ of arc) with the Master Ortho-Rater (Bausch \& Lomb).

Apparatus and Stimuli. PC-controlled PLATO liquid crystal shutter glasses (TNO Institute for Perception, Soesterberg, The Netherlands; transmittance of the open glass, $85 \%$; effective turnon and turn-off time, $<2 \mathrm{msec}$; see Milgram, 1987, for further details) were used to manipulate the viewing conditions. Stereoacuity (minimal disparity) was measured with a three-rod apparatus. This apparatus had a frontage size of $600 \times 450 \mathrm{~mm}\left(11.3^{\circ} \times 8.5^{\circ}\right.$ visual angle), including a window of $280 \times 60 \mathrm{~mm}\left(5.3^{\circ} \times 1.2^{\circ}\right)$. The rods (small black glass tubes) were $2 \mathrm{~mm}$ ( $2.3 \mathrm{~min}$ of arc) thick and separated by $75 \mathrm{~mm}\left(1.4^{\circ}\right)$. The window's height $\left(60 \mathrm{~mm}, 1.2^{\circ}\right)$ limited the visible length of the rods and kept it constant. The distance between the participant's eyes and the rods was $3 \mathrm{~m}$. The frontage had a luminance of $300 \mathrm{~cd} / \mathrm{m}^{2}$, and the background luminance was $400 \mathrm{~cd} / \mathrm{m}^{2}$.

In the three-rod apparatus, three parallel vertical rods are observed simultaneously. The two outer rods are stationary, whereas the middle one may be moved in the sagittal plane (with respect to the observer) either toward or away from the observer. If the shift is sufficiently large, the middle rod can be seen in front of or beyond the plane defined by the two outer rods (Helmholtz, 1910).

In this apparatus, in principle, four monocular cues are available: (1) motion parallax, which, however, was eliminated by the use of a headrest; (2) the varying of the visual angle of the middle rod's length with the rod's movement toward or away from the observer, which, however, was made invisible to the observer by the window of constant height in front of the rods; (3) the varying of the visual angle of the middle rod's width with the rod's movement toward or away from the observer by $\pm 4.7 \mathrm{sec}$ of arc, which is clearly below threshold; and (4) the rods' relative distances in monocular viewing (e.g., for the right eye the leftmost two rods appear closer together when the middle rod is in front of the two outer ones). This cue, however, should not play a role in our experiments, since Westheimer and McKee (1979) showed that, with distances among three lines of more than $5 \mathrm{~min}$ of $\operatorname{arc}\left(1.4^{\circ}\right.$, or $84 \mathrm{~min}$ of arc in our arrangement), the threshold for depth discrimination based on stereoscopic information (disparity detection) is clearly lower than the threshold based on the apparent distance between the lines, which is a monocular cue. In other words, monocular cues are very likely to play no role with only three lines in an arrangement such as ours.

Procedure. To measure stereo thresholds, we used the classic psychophysical method of limits (see, e.g., Woodworth \& Schlosberg, 1964), in which ascending series (in which the distance between the middle rod and the outer rods is increased until the observer detects the depth) and descending series (in which the distance is reduced until the observer sees no more depth) alternate. Both directions of movement of the middle rod - toward and away from the observerwere used. In each of these cases, the middle rod was moved continuously with a speed of $10 \mathrm{~cm}$ per $30 \mathrm{sec}$ - that is, $1.9^{\circ}$ per $30 \mathrm{sec}$, or approximately $4 \mathrm{sec}$ of arc per second. For each movement direction, an ascending and a descending series were always presented in alternation, whereas the order of the directions of movement was randomized. The threshold distance and the individual interocular distance were used to compute an observer's minimum recognizable disparity (threshold disparity), and the resulting disparities were averaged across both movement directions and across descending and ascending series in order to compute stereoacuity.

Experiment 1 consisted of four conditions for measuring stereoacuity.

Control conditions. As a control of the shutter glasses' influence in general, an observer's stereoacuity was measured without shutter glasses (Control Condition A) and using shutter glasses with both eyes open (Control Condition B). The order of Control Conditions A and $\mathrm{B}$ was randomized across observers.

Frequency condition. Using shutter glasses, stereoacuity was determined for presentation frequencies of 1, 2, 4, 8, 16, and $32 \mathrm{~Hz}$ without interocular delay and with the six frequencies presented in random order. An alternation frequency of $1 \mathrm{~Hz}$, for instance, means that the left eye's monocular image is presented for $500 \mathrm{msec}$, followed by presentation of the right eye's monocular image for $500 \mathrm{msec}$ (without temporal overlap of the two images and with no delay between them), followed again by presentation of the left eye's image for $500 \mathrm{msec}$, and so on.

Delay condition. Using shutter glasses, stereoacuity was determined with exposure times of the monocular images fixed at either 25 or $50 \mathrm{msec}$ and with interocular delays (between the offset of one 
image and the onset of the next) of 0,25 , or $50 \mathrm{msec}$, presented in random order. The resulting stimulation frequencies are presented in Table 1.

The order of the conditions (alternating between observers) was either control-frequency-delay or control-delay-frequency.

\section{Results and Discussion}

Figure 1 shows the resulting mean stereoacuity obtained in the frequency condition and in the delay condition as a function of the monocular alternating frequency, as well as the mean stereoacuity obtained in the control conditions with open shutter glasses and without shutter glasses. We will first consider the effect of the monocular alternating frequency on the stereoacuity in the frequency condition - that is, without interocular delay.

Stereoacuity increased (and stereo thresholds decreased) with increasing frequencies from $90 \mathrm{sec}$ of arc at $1 \mathrm{~Hz}$ to $39 \mathrm{sec}$ of arc at $4 \mathrm{~Hz}$. There was a smaller increase of stereoacuity from $4 \mathrm{~Hz}(39 \mathrm{sec}$ of arc) to $16 \mathrm{~Hz}(18.5 \mathrm{sec}$ of arc). Little further improvement was found for a frequency of $32 \mathrm{~Hz}$. We compared each threshold value at a given frequency with that of the neighboring frequency by $t$ tests. With the Bonferroni adjustment of degrees of freedom, all of these differences were significant [all $t \mathrm{~s}(14)>$ 3.95, all $p$ s $<.002]$.

In the literature, it is often claimed that the limit for stereo vision is a 100 -msec duration per eye, which corresponds to the frequency of $5 \mathrm{~Hz}$. This frequency resulted in a stereoacuity of approximately $35 \mathrm{sec}$ of arc, which is about half as good as that found in the control conditions. This limit, therefore, appears more or less arbitrary if one considers the threshold's course in Figure 1.

We used function fit to describe how the stereo threshold in the frequency condition depends on alternating frequency. If frequency is plotted on a logarithmic scale as in Figure 1, the threshold follows an exponential function with the equation $y=89.32 * \mathrm{e}^{(-1.26 * x)}\left(r^{2}=.98\right)$. This corresponds to the threshold following a power function with the equation $y=89.32 * x^{-0.55}\left(r^{2}=.98\right)$ if the frequency is plotted on a nonlogarithmic scale.

Let us compare the results of our frequency condition with those of the two earlier studies in which stereoacuity with alternating monocular images was measured (Ogle, 1963; Wist \& Gogel, 1966). Ogle reported decreasing thresholds with increasing exposure times of the monocular images. At first glance, this contradicts our result of decreasing thresholds with increasing frequencies (i.e., decreasing exposure times of the monocular images). This apparent discrepancy, however, is due to the fact that in Ogle's study increasing exposure times were confounded

Table 1

Stimulation Frequencies (in Hertz) As a Function of Exposure Time and Interocular Delay in the Delay Condition of Experiment 1

\begin{tabular}{ccc}
\hline \multirow{2}{*}{$\begin{array}{c}\text { Interocular } \\
\text { Delay }(\mathrm{msec})\end{array}$} & \multicolumn{2}{c}{ Exposure Time } \\
\cline { 2 - 3 } & $25 \mathrm{msec}$ & $50 \mathrm{msec}$ \\
\hline 0 & 20 & 10 \\
25 & 10 & 6.66 \\
50 & 6.66 & 5 \\
\hline
\end{tabular}

with an increasing temporal overlap between the two images. If conditions with equal (or similar) delays between the two monocular images are considered, then longer exposure times (corresponding to lower frequencies) resulted in increased thresholds, as in our Experiment 1. Thus, our results confirm those of Ogle's study, but without confounding. Wist and Gogel did not vary the duration of the monocular images but only the delay within and between successive pairs of images. In each case they used an interocular delay, in contrast to our frequency condition. We will, therefore, compare their findings with ours in the discussion of the results in our delay condition.

In Control Condition A, with binocular viewing without shutter glasses, a mean stereo threshold of $16 \mathrm{sec}$ of arc (which is within the normal range) was reached. Viewing through the shutter glasses with both eyes open in Control Condition B degraded stereoacuity only slightly, but significantly $[t(14)=2.74, p<.02]$, to a value of $20 \mathrm{sec}$ of arc. The monocular viewing conditions used in Experiment 1 resulted in even slightly better values of spatial acuity of less than $20 \mathrm{sec}$ of arc minimal disparity at high alternating frequencies (16 Hz or more). Perhaps this may be accounted for by perceptual learning, since the control conditions were always carried out at the beginning of the experiment. Perceptual learning - that is, the improvement of perceptual performance with training or across repeated measures - has already been shown for stereoacuity (see, e.g., Fendick \& Westheimer, 1983; Wallach, Moore, \& Davidson, 1963).

The results from the delay condition shown in Figure 1 allow for a direct comparison with the results obtained in the frequency condition. (The exposure times and corresponding frequencies for the delay condition have been given in Table 1.) In the 0 -msec delay condition, the presentation times of 25 and $50 \mathrm{msec}$ correspond to frequencies of 20 and $10 \mathrm{~Hz}$, respectively. At $10 \mathrm{~Hz}(50 \mathrm{msec})$, the delay condition resulted in a threshold of about $25 \mathrm{sec}$ of arc, whereas interpolating between the values for 8 and $16 \mathrm{~Hz}$ from the frequency condition leads to about $23 \mathrm{sec}$ of arc. At $20 \mathrm{~Hz}(25 \mathrm{msec})$, a threshold of about $20 \mathrm{sec}$ of arc was found, whereas interpolating between the values for 16 and $32 \mathrm{~Hz}$ leads to about $17 \mathrm{sec}$ of arc. Thus, the two values obtained in the $0-\mathrm{msec}$ delay condition are slightly larger than the corresponding values interpolated from the frequency condition. Perhaps these small differences are accidental, but they also may be due to the fact that the context of interocular delays in this condition led to worse binocular viewing and higher thresholds.

Figure 1 further shows that with an interocular delay of $25 \mathrm{msec}$, stereo thresholds clearly are higher than those obtained in the frequency condition with corresponding frequencies but with no interocular delay. With an interocular delay of $50 \mathrm{msec}$, thresholds are even higher. Interestingly, the courses of thresholds obtained with delays of 25 and $50 \mathrm{msec}$ seem to run parallel to that obtained without delay.

Figure 2 illustrates these results for a more detailed analysis of the effects of exposure time and delay in the delay condition. Increasing interocular delays between the monocular exposures from 0 to $50 \mathrm{msec}$ led to de- 


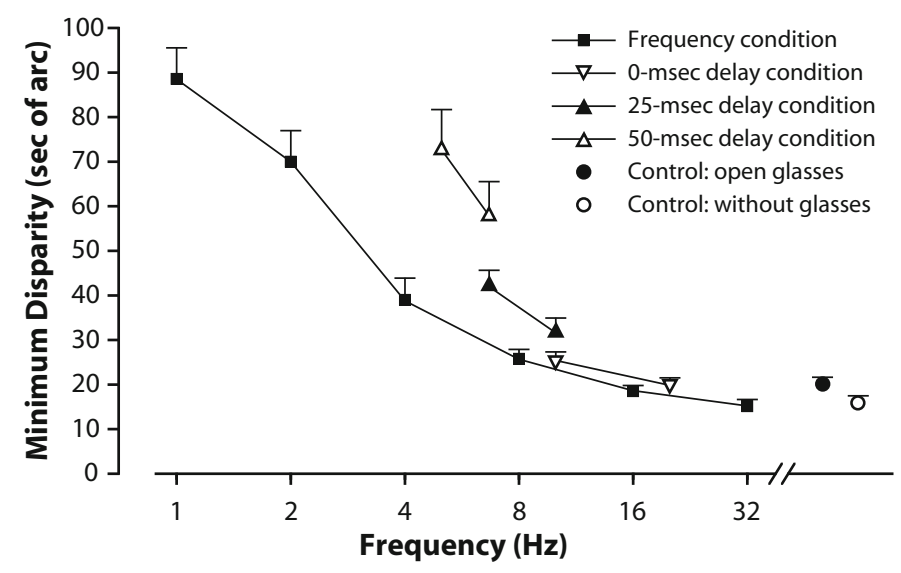

Figure 1. Mean stereoacuity (minimum disparity, in seconds of arc) and standard errors in the frequency and delay conditions as a function of monocular alternating frequency, and in the control conditions with open shutter glasses and without shutter glasses.

creasing stereoacuity (increasing thresholds). The effect of exposure time corresponded to the frequency effect as measured in the frequency condition: The longer exposure time, corresponding to a lower presentation frequency, resulted in a higher minimum disparity. Both effects were confirmed in an ANOVA with two within-subjects factors: exposure time ( $25 \mathrm{vs.} 50 \mathrm{msec}$ ) and interocular delay $(0$ vs. 25 vs. $50 \mathrm{msec})$. In this analysis, the main effects of exposure time $[F(1,14)=16.34, p<.002]$ and interocular delay $[F(2,28)=32.14, p<.001]$ were significant, whereas the interaction between the two factors was not $[F(2,28)=1.11, p>.31]$.

We will now compare our results from the delay condition with those of earlier studies. Herzau (1976) reported that with decreasing stimulus durations (or exposure times), increasingly longer delays between the two monocular images were tolerated without loss of stereopsis. Our results confirm Herzau's finding if we look at some particular stereo threshold instead of at the loss of stereopsis in an all-or-none fashion. For example, in Figure 2, note the threshold of $25 \mathrm{sec}$ of arc for both exposure times. This threshold value was reached with the longer exposure time $(50 \mathrm{msec})$ at an interocular delay of $0 \mathrm{msec}$, but the same threshold was reached with the shorter exposure time $(25 \mathrm{msec})$ at an interocular delay of about 10 or $15 \mathrm{msec}$. Also, for any other threshold value the shorter exposure time corresponds to a longer delay. Thus, our delay results for stereoacuity correspond well with Herzau's findings for stereopsis per se.

Ross and Hogben (1974) found that stereopsis got increasingly worse with increasing delays between the two monocular images. This finding also corresponds well with our result of increasing thresholds (or decreasing stereoacuity) with increasing interocular delays (for both exposure times). Furthermore, our results confirm the findings of Ogle (1963) and of Wist and Gogel (1966),

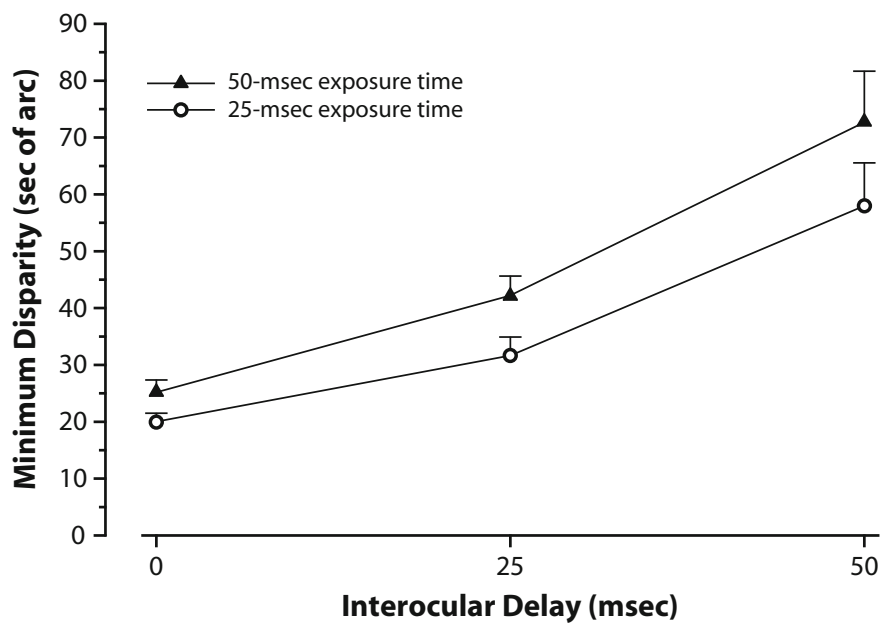

Figure 2. Mean stereoacuity (minimum disparity in seconds of arc) and standard errors in the delay condition as a function of monocular exposure time and interocular delay. 
who reported increasing stereo thresholds with increasing interocular delays. The results reported so far are also in good correspondence with a pilot study (Pieper \& Ludwig, 1998) with almost identical conditions, aside from the use of shutter glasses with lower transmittance.

\section{EXPERIMENT 2}

Experiment 1 demonstrated the usefulness of electronically controlled shutter glasses in studying stereovision with alternating monocular images. In contrast to earlier methodological approaches, this new method allows for the independent control of frequency and interocular delay. Hence, in Experiment 2 we could use this method to study whether or not binocular phenomena other than stereopsis may also be generated with alternating monocular images. We chose to study binocular luster (instead of, e.g., binocular rivalry) to answer this question, mainly because (1) binocular luster, like luster in general, can be observed in everyday life and (2) luster is a very stable impression, like color impressions, for example. Stereopsis and binocular luster are the two binocular phenomena that occur under normal viewing conditions and in which information is gained from differences between the two retinal images. Stereopsis provides information on depth and, thus, on the shapes and locations of objects; luster relates to a characteristic of object surfaces - namely, whether they are smooth or rough. Binocular luster plays a key role in the perception of surfaces, especially metallic ones, and makes objects strangely conspicuous among other objects (see, e.g., McCamy, 1998). Therefore, it is quite different from the impression of binocular rivalry, which is a very unstable impression and is not seen under everyday viewing conditions.
Binocular luster emerges when an object is observed that presents different levels of luminance to the two eyes. Under experimental conditions, it is elicited when, under binocular viewing, two patterns (e.g., circles) of the same shape and size but of different luminances are presented to the two eyes and fused. Disk B in Figure 3 illustrates these conditions. In this case, the two outer rims may be fused and appear as a black rim, whereas the inner parts of the discs appear as a homogenous shimmering or silvery sheen called "binocular luster." Binocular rivalry, by contrast, can be perceived when two completely different images, such as a dark disk and a light disk or a red disk and a green disk, are presented to the two eyes and must be fused. This results in the impression that one of the two luminances or colors dominates over the other for a limited time and afterward the other one dominates; for example, the disk alternately looks light and dark or red and green. With relatively large monocular images differing, for example, in luminance or in color, rivalry occurs as well, but then some parts of the field appear in the luminance or color of one image and other parts in the luminance or color of the other image (so-called mosaic rivalry; see Howard \& Rogers, 1995). (For a detailed description of the phenomena of binocular luster and rivalry, see Helmholtz, 1910.)

As an ancillary goal of Experiment 2, we attempted to compare (1) stereopsis of a Julesz (1971) random-dot stereogram and (2) binocular luster, with respect to the minimum alternating frequency of the two half-images and the maximum interocular delay that are tolerated without a breakdown of the impression. This comparison could be relevant for understanding the mechanisms underlying binocular vision under alternating monocular stimulation. Up until now, only stereopsis resulting from two monocu-

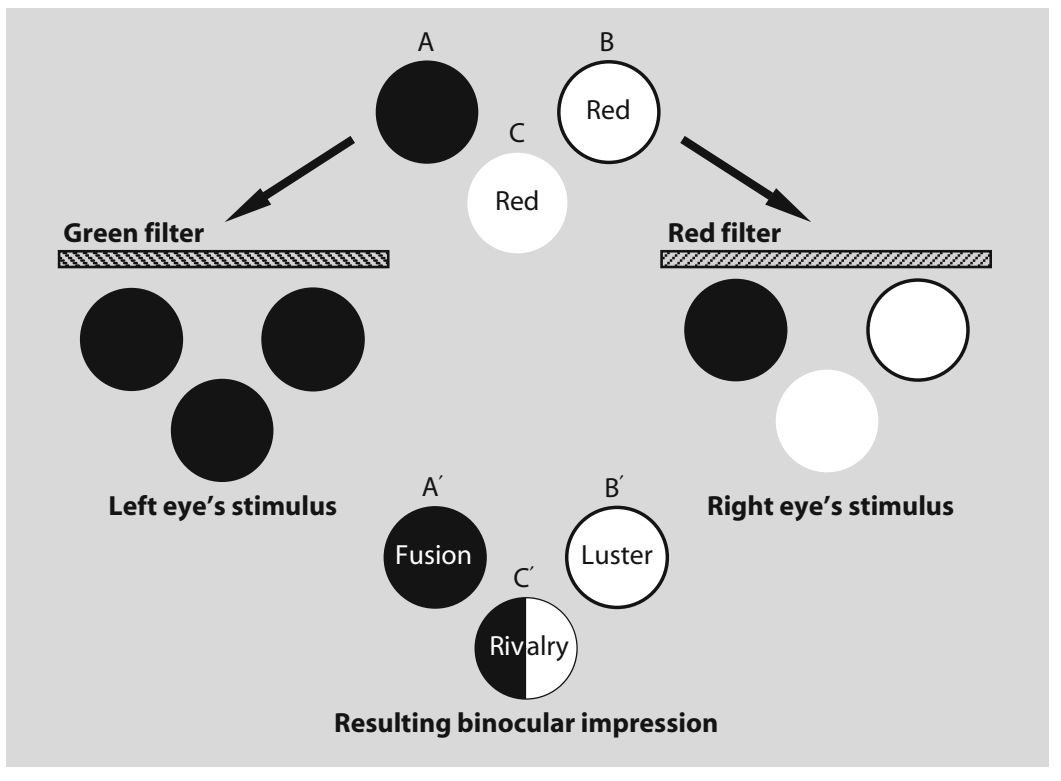

Figure 3. Arrangement of three disks (A, B, and C) similar to those of Levelt (1965), the green and red anaglyphs for the left and right eyes, and the binocular impression. $\left(A^{\prime}\right)$ binocular fusion; ( $\left.B^{\prime}\right)$ binocular luster; $\left(C^{\prime}\right)$ binocular rivalry. 
lar images containing binocular disparity was considered. We will, however, include binocular luster as one other binocular phenomenon in which the monocular images do not contain binocular disparity.

Figure 3 illustrates the stimulus conditions for binocular luster. The two halves of three disks are presented stereoscopically using red and green anaglyphs. Disc A, with the two identical monocular halves, is binocularly fused $\left(\mathrm{A}^{\prime}\right)$. Disc $\mathrm{C}$, with two different halves, underlies binocular rivalry $\left(\mathrm{C}^{\prime}\right)$. Rivalry within a small area shows exclusive dominance - that is, either one or the other image is seen exclusively at any time. In Disc B, the outer rims may be fused whereas the inner parts have rival content. When corresponding regions of the two eyes are stimulated by different luminous patterns (and the size of the discs exceeds $1^{\circ}$ - see Pieper \& Ludwig, 2001), a special binocular impression, called binocular luster, can be observed. Thus, in Disc B the inner part of the disc appears as a silvery sheen, or binocular luster $\left(\mathrm{B}^{\prime}\right)$. For details, see also Levelt (1965).

\section{Method}

Participants. Thirteen University of Giessen students of psychology (8 men and 5 women) took part in Experiment 2. Their mean age was 23.5 years (range, 20-27 years). They received course credit for their participation. All the participants were tested for normal visual acuity and normal stereoscopic acuity (at least $19 \mathrm{sec}$ of arc) with the Master Ortho-Rater (Bausch \& Lomb).

Apparatus and Stimuli. Since the PC-controlled LCD shutter glasses used in Experiment 1 were not available, we used an older version (3D-Max, Kasan Electronics) to manipulate the viewing conditions. In these glasses, the open LCD shutter has a lower transmittance (30\%), but this proved not to be critical for the variables in question. Stimuli for the two stereopsis conditions (see below) were the anaglyphs of a random-dot stereogram in red and in green on a white background. Stimuli for the two luster conditions (see below) were the anaglyphs of an arrangement of three dots, as illustrated in Figure 3, again on a white background. Both the red and the green dots had a luminance of $60 \mathrm{~cd} / \mathrm{m}^{2}$, and the white background had a luminance of $100 \mathrm{~cd} / \mathrm{m}^{2}$. The red and green filters (used to separate the images by anaglyph stereoscopy) had transmittances of $36 \%$ and $26 \%$, respectively. The stereogram had a $300-\mathrm{mm}\left(16.7^{\circ}\right)$ lateral length and 25 min of arc disparity. The dot diameter in the luster stimuli was $37 \mathrm{~mm}\left(2.1^{\circ}\right)$, and the arrangement of the three dots had a width of $177 \mathrm{~mm}\left(10.1^{\circ}\right)$ and a height of $107 \mathrm{~mm}\left(6.1^{\circ}\right)$. The stimuli were projected onto a screen at a viewing distance of $1 \mathrm{~m}$ in both conditions.
Procedure. There were four conditions in Experiment 2: stereogram-frequency, stereogram-delay, luster-frequency, and luster-delay. In the two stereogram conditions, the anaglyphs of a random-dot stereogram were presented, and the participants had to indicate whether or not they could see the global figure (a diamond) in it. In the two luster conditions, the anaglyphs of the stimulus shown in Figure 3 were presented, and the participants had to indicate the impression of luster in the upper right dot. The other two dots, one of which resulted in fusion and the other in rivalry, were used to ensure that fusion was stable and that the observers could differentiate these impressions from the impression of luster. Using the method of limits, in the two frequency conditions (without interocular delay), the frequency of the monocular images was increased (ascending series) or decreased (descending series) until the perception of the target (global figure or luster, respectively) changed from "not present" to "present" or vice versa. In the two delay conditions, the same procedure was applied to the delay between the monocular images, with the presentation time of each monocular image fixed at $25 \mathrm{msec}$. Five descending and five ascending series followed each other alternately within each condition. The order of the four conditions was permutated across the participants. Thresholds were computed separately for each condition and each observer as the mean value of the corresponding ascending and descending series.

\section{Results and Discussion}

Let us now compare the results of the frequency and delay conditions between stereopsis and binocular luster. As was mentioned above, both are binocular phenomena, but only in stereopsis does binocular disparity play a role, whereas in binocular luster the stimuli are not disparate. If the integration of the two monocular images is based on mechanisms that specifically process binocular disparity, then this integration should be possible in stereopsis but not in binocular luster. If, however, more general binocular mechanisms are responsible for this integration, then integration of monocular images separated in time should be possible in both stereopsis and luster.

Figure 4 shows the results for the two frequency conditions (left panel) and the two delay conditions (right panel) for stereopsis and binocular luster. First, both phenomena occurred under successive monocular stimulation. We found a breakdown frequency of $3 \mathrm{~Hz}$ for seeing a global figure in a random-dot stereogram, which corresponds to a $167-\mathrm{msec}$ exposure time of the anaglyph for each eye. Below that frequency, no stereopsis was achieved. This corresponds well with Engel (1970), who also used
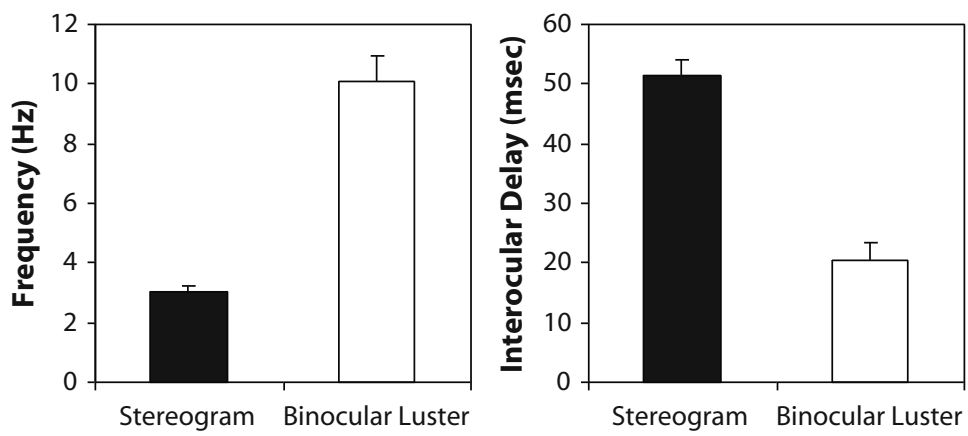

Figure 4. Mean (with standard errors) of the frequency thresholds (left panel) and the thresholds of interocular delay (right panel) for detecting a global figure in a random-dot stereogram and binocular luster. 
random-dot stereograms as stimuli. He found that stereoscopic depth was visible with stimulus repetition rates between 2.5 and $10 \mathrm{~Hz}$.

In contrast, binocular luster was observed only with frequencies more than three times higher (about $10 \mathrm{~Hz}$ ), corresponding to an exposure time of the monocular images of $50 \mathrm{msec}$. The difference between the frequency thresholds for stereopsis and for binocular luster was significant [for paired samples, $t(12)=-7.53, p<.001$ ].

Thus, at least for the specific stimuli and conditions that we used in Experiment 2, stereoscopic depth perception tolerates a much lower frequency of alternating monocular presentations than does the phenomenon of binocular luster. Inspection of the individual data showed that the direction of the differences was the same for all the observers, although their amount varied. Between the observers' individual frequency thresholds for seeing stereoscopic depth or binocular luster, there was no correlation (Kendall's $\tau=-.01, p=.95$ ). The mean threshold of interocular delay was found to be about $51 \mathrm{msec}$ for detecting the global figure in a random-dot stereogram, but about $20 \mathrm{msec}$ for binocular luster. Again, the difference between the thresholds for stereopsis and binocular luster was significant [for paired samples, $t(12)=10.85, p<$ $.001]$, and the individual differences had the same direction for all the observers. As for the frequency thresholds, there was no significant correlation between the observers' individual delay thresholds for seeing stereoscopic depth and their delay thresholds for seeing binocular luster $(\tau=$ $.15, p=.46$ ). On the whole, with respect to frequency as well as to delay, temporal integration was significantly better for stereopsis than for binocular luster.

\section{GENERAL DISCUSSION}

Both experiments show that the PC-controlled shutter glasses we used to produce a stimulation with alternating monocular images are well suited to create stereoscopic vision and for the study of its specific quantitative characteristics. The use of those instruments allows one to manipulate the relevant variables (e.g., monocular presentation times, interocular delay) very accurately and independently both of each other and of further stimulation variables (e.g., the intensity of the monocular images). In comparison with the methods used in earlier studies, this is of great advantage. Experiment 2 showed that binocular luster, another binocular phenomenon, may also be obtained under conditions of alternating monocular stimulation. As far as we know, this is the first time this has been shown.

Our results in general confirm those of Dvorák (1872), Exner (1875), Guilloz (1904), and Ewald and Gross (1906) with respect to the point that stereopsis may emerge with alternating stimulation by monocular images. In contrast, Stevenson and Sanford (1908) did not find an interval that could be inserted between stimuli successively presented to the two retinas without destroying a stereoscopic relief. In light of both our results and some of those described in the introduction, however, their negative result is not surprising because their alternation frequency was $1.25 \mathrm{~Hz}$ instead of the minimum of about $3 \mathrm{~Hz}$. Thus, the finding that stereoscopic vision can result from the sequential or alternating presentation of monocular images (provided that the presentation frequency of the two half-images is high enough) may be considered widely accepted. Even short delays between the two monocular stimuli are bridged if they follow each other in rapid succession.

Some authors (e.g., Howard \& Rogers, 1995) suggested that this might be due in part to afterimages of the monocular stimuli, which then interact with the subsequent stimuli. Ewald and Gross (1906), however, separated the monocular images by glaring color spots, which let the afterimage vanish before the other eye had seen its image. Nevertheless, a stereoscopic impression emerged. Ogle (1963) reported that Ogle and Reiher (1962) found that strong afterimages induced in the two eyes successively can indeed result in stereoscopic depth perception. The stereoscopic impression, however, faded rapidly even though the afterimages themselves lasted much longer. Finally, in our pilot study (Pieper \& Ludwig, 1998), the luminance of the stimuli was very low, so that afterimages presumably were not relevant. On the whole, afterimages of monocular stimuli at least are not a prerequisite for stereopsis under these conditions.

Another widespread explanation of stereopsis under successive monocular stimulation assumes that some "remnant" of the first stimulus (e.g., a "recollected image" in the terminology of Ewald \& Gross, 1906; "information" or a "monocular response," as Engel, 1970, put it; or a "memory trace," in the words of Ross \& Hogben, 1974) outlasts the stimulus itself and thus may be integrated with the second stimulus. This surely is a reasonable assumption because there are many other examples, such as flicker fusion and apparent movement, in which delays between successive stimuli are "bridged" by the nervous system. Because such storage is limited in time, stereopsis should break down with frequencies of alternation that are too low and empty intervals between the monocular stimuli that are too long. If this rather general explanation is true, however, it should hold for different examples of binocular vision. O'Shea and Crassini (1984) reported that binocular rivalry could be observed with alternating frequencies as low as $2 \mathrm{~Hz}$ and that it tolerated delays of $100 \mathrm{msec}$ between 5 -sec monocular presentations. Our Experiment 2 showed that binocular luster also appears under conditions of alternating monocular stimulation. This suggests that a rather general process is indeed responsible for the integration of monocular images separated in time.

However, our Experiment 2 also showed that the temporal parameters for binocular luster and stereopsis were quite different, although they were measured under very similar conditions and in the same participants. Binocular luster broke down at frequencies and with delay durations at which stereopsis still worked. Furthermore, we found no significant correlation at all between the individual thresholds for perceiving stereoscopic depth and those for perceiving binocular luster, when either the frequency threshold or the delay threshold was considered. This could suggest that different processes (with different 
temporal requirements) are involved in temporal integration in the cases of stereopsis and binocular luster. Nevertheless, since in Experiment 2 we compared the results from only one stereogram and one luster stimulus, one could argue that the differences in the critical alternating frequency and in the critical interocular delay may be due to the particular characteristics of the stimuli we used (e.g., disparity and patch size of the luster stimulus) rather than to the processes involved. We took care, however, that the stimuli for stereopsis and for binocular luster were quite comparable. Therefore, in Experiment 2 we did not use a stereo stimulus as the three-line stimulus from Experiment 1, since such stimuli are not suitable for creating luster. Both stimuli in Experiment 2 were surfaces of the same color and similar luminances, viewed with anaglyphs. Furthermore, in the luster stimulus, the dot diameter may not be varied without limits because dots that are too small produce the sieve effect (Howard, 1995) - a depth impression without disparity - and dots that are too large produce binocular rivalry (Pieper \& Ludwig, 2001). Finally, it is impossible to make the two stimuli comparable with respect to disparity, since the luster stimulus does not contain disparity.

Thus, in future experiments, instead of trying to make both stimuli even more comparable than we did, researchers should examine the extent to which the critical alternating frequency and the critical interocular delay are influenced by stimulus parameters. This would help to decide whether the differences in temporal integration for stereopsis and for binocular luster that we found are due to the characteristics of the specific stimuli we used or to different underlying processes. If it turns out that different processes are involved, one could ask what these might be. At present, we can only speculate.

On the one hand, it is known that binocular cortical neurons exist that are sensitive to certain binocular disparities (see, e.g., Barlow, Blakemore, \& Pettigrew, 1967; Pettigrew, Nikara, \& Bishop, 1968). In the cases of measuring stereo thresholds (Experiment 1) and global stereopsis (Experiment 2, stereo condition), such neurons might have been involved in detecting depth. Perhaps such neurons also accomplish the temporal integration of two disparate monocular images separated in time. On the other hand, very little is known about the physiological basis of the perception of binocular luster. We assume that binocular luster arises from the activity of particular neurons with binocular receptive fields that are activated by a binocular stimulation with negatively correlated luminance values (i.e., low values in one eye and high values in the other) in the same part of the visual field. Perhaps neuronal structures dealing with the processing of luminance and contrast contribute to this phenomenon. In any case, the monocular images resulting in binocular luster in Experiment 2 did not contain any disparity. Therefore, in contrast to the case of stereopsis, neurons with binocular receptive fields but without sensitivity for disparity may be involved in the detection of binocular luster. Nevertheless, according to our psychophysical results, the neurons involved in the perception of binocular luster accomplish the temporal integration of monocular images too, but to a lesser extent than in the case of stereopsis. Hopefully, the present psychophysical results may serve as a starting point for physiological attempts to identify the (different) processes and structures.

Finally, we will look at phenomena related to those we studied here: (1) the so-called monocular luster in its relation to binocular luster, and (2) the Pulfrich effect, Pulfrich-like effects, and the Mach-Dvorák effect in relation to stereopsis from alternating monocular stimulation.

Anstis (2000) reported that a spot flickering at $16 \mathrm{~Hz}$ between two luminances on a gray surround had an appearance of metallic luster similar to the well-known binocular luster. He called this phenomenon monocular luster. However, since the flickering spot was presented to both eyes at the same time and viewed binocularly, in our opinion the term monocular luster might be misleading and therefore should be replaced by flicker luster or a similar term. Anstis asked his observers to rate the subjective luster of a spot flickering between two luminances (monocular luster) and that of two binocularly fused spots of differing luminances (binocular luster) and varied the luminances of the spots. He found that the lustrous appearance of the spots in both the monocular and binocular conditions was strongest when the two luminance values straddled the luminance value of the surroundings. As Anstis pointed out, it is the contrast reversal of the spot (between the spot's luminance and the luminance of the surroundings) that makes it appear lustrous in both the monocular and binocular conditions. Anstis regards monocular luster as the temporal analog of binocular luster, since both occur under similar conditions when opposite luminance polarities are combined either across eyes or over time. He assumes that a flickering spot stimulates an "on" pathway while in its spatial increment phase (i.e., while its luminance exceeds the surround luminance) and a separate "off" pathway while in its spatial decrement phase (i.e., while its luminance is lower than the surround luminance) and attributes monocular luster to rival competition between the "on" and the "off" pathways.

In light of the results of our Experiment 2, however, new questions concerning so-called monocular luster arise. In Anstis's (2000) monocular luster condition, two spots (one for each eye) simultaneously flickered between a high and a low luminance at a rate of $16 \mathrm{~Hz}$. In the luster condition of our Experiment 2, a light spot was presented to one eye and a dark spot to the other. Instead of simultaneous presentation, however, the spots appeared subsequently, alternating at varying frequencies. With frequencies of at least $10 \mathrm{~Hz}$, this stimulation resulted in the appearance of (binocular) luster. One could imagine that the same thing occurred in Anstis's monocular luster condition as in our luster condition: Each dark spot in one eye might have been binocularly fused with the following light spot in the other eye, resulting in the appearance of luster. According to our results, the frequency used by Anstis $(16 \mathrm{~Hz})$ is sufficient to allow for such temporal integration between the eyes. Thus, Anstis's phenomenon of so-called monocular luster might in fact be binocular luster emerging from binocular temporal integration. The important question now is whether or not a flickering spot such as that used by 
Anstis would also appear lustrous if it were viewed monocularly. If this is the case, it would indeed be a monocular phenomenon quite different from the binocular luster we observed. Then, it might result from the activity in neurons with monocular inputs, whereas the binocular luster we observed requires neurons with binocular inputs.

Let us close by considering that, at first glance, the phenomenon of stereopsis caused by alternating monocular stimulation might be similar to the so-called Pulfrich effect (Pulfrich, 1922). Here, a pendulum swinging in a frontal parallel plane is viewed with both eyes while one eye is covered by a neutral density filter. The pendulum then appears to swing along an elliptical orbit. This effect is usually explained by the fact that the weaker stimulus (the reduced amount of light) at the covered eye results in a temporally delayed neuronal transmission from that eye's retina to the cortex. Thus, since the positional signal of the pendulum varies in time, on the cortical level the signals from both eyes include a disparity that results in perception of the apparent depth in the pendulum's orbit. This explanation is supported by Pulfrich-like effects in which dimming the view of one eye was replaced by directly delaying it (see, e.g., Burr \& Ross, 1979). Thus, in the Pulfrich effect apparent depth results from interocular delay (at the cortical level), although the target (the swinging pendulum) does not include any "real" disparity in time. Therefore, this effect is only superficially related to stereopsis from alternating monocular stimulation, since in the latter case the two monocular images per se include real disparity and are presented to the eyes only successively. This successive presentation does not cause additional apparent disparity as in the Pulfrich effect, since in our case the target does not change its position by moving.

Interestingly, Julesz and White (1969) tested the classical explanation for the Pulfrich effect - the so-called delay-line hypothesis - and stumbled across stereopsis with successive monocular stimulation. As was mentioned above, according to this hypothesis, filtering in one eye in effect puts a delay line in the system for that eye. Julesz and White prepared a film loop consisting of a sequence of random-dot stereograms in which a square could be seen in front of the surrounding dynamic noise when viewed binocularly. Now, the stereo pairs were displaced by one frame - that is, frame $n$ for one (the "leading") eye was presented simultaneously with frame $n-1$ for the other ("lagging") eye. When viewing this film loop with a filter over the leading eye, an observer should report stereoscopic depth since the lead in the film loop should be compensated by the filter-induced delay. This is what Julesz and White found, which supports the classical Pulfrich explanation. Also in accordance with this explanation, at lower film speeds the filter was adjusted to greater density than it was at faster speeds. In addition to this, and unexpected by Julesz and White, however, at faster film speeds ( $\geq 19$ frames/sec) stereopsis was obtained without filtering. From our point of view, and in light of the studies mentioned in the introduction, this finding is not surprising, but Julesz and White seem to be the first to have observed stereopsis with successive monocular stimulation with random-dot stereograms, preceding
Engel (1970) and Ross and Hogben (1974). In explaining their findings, Julesz and White assumed that the stereoscopic depth mechanism has an inertia with a time constant during which successive film frames might be retained and averaged. At fast film speeds, many frames of the left and right matrices, including many binocularly correlated frames that permit stereopsis, are averaged. But at slow film speeds, only a few frames are averaged and the averages of the left and right matrices have inadequate correlation for stereopsis to occur. Taking into account additional findings from apparent movement studies led Julesz and White to the assumption that stereopsis (the localization of binocular information in depth) and the cyclopean view (the binocular combination of the two eyes' views into a single percept) are two different mechanisms, and the time constant for stereopsis might be larger than that for binocular combination. This assumption might be interesting with respect to the results of our Experiment 2. If it turned out that the different results for stereopsis and binocular luster were not bound to the particular stimulus displays we used, perhaps they could be accounted for by two different mechanisms with different time constants, as Julesz and White assumed.

Note that in studies of the Pulfrich effect (e.g., Morgan, 1979, 1981; Morgan \& Ward, 1980), sampled motion, in which the eyes are never simultaneously stimulated, was also used. We think, however, that the detailed description and discussion of these studies would go beyond the scope of this article. For recent work on the Pulfrich and Pulfrich-like effects, see, for example, Morgan and Castet (1995), Qian and Anderson (1997), and Morgan and Fahle (2000). Morgan and Castet (1995) and Morgan and Fahle (2000) suggested that phase lag rather than delay per se is the critical factor in stereopsis with periodic stimuli.

We end with a brief look at the Mach-Dvorák effect (Dvorák, 1872) and its relation to our experiments. In this phenomenon, a pendulum swinging along a horizontal path is viewed through an episcotister, which presents alternating views to the two eyes or is illuminated intermittently, with exposures at slightly different times to the two eyes. As in the Pulfrich effect, the pendulum then appears to swing on an elliptical path in depth. In the Mach-Dvorák effect, however, the apparent depth does not result from differential filtering of the target to the two eyes but from a retinal disparity produced directly by the time delay in stimulus presentation to each eye. The visual system thus integrates signals arriving from the two eyes at different times. Morgan (1979) argued that the integration process has a limited temporal range that is not sufficiently long to include events $50 \mathrm{msec}$ apart. Thus, the Mach-Dvorák effect is another example of the fact that stereopsis does not break down with interocular delays. Our Experiment 1 led to the same conclusion but, in addition, showed that stereo acuity gradually got worse with increasing interocular delays. We did not find a fixed time constant with an abrupt breakdown of stereopsis (on-off effect) but a gradual deterioration with increasing interocular delays. Our Experiment 2 further showed that not only stereopsis but also binocular luster tolerates delays between monocular signals. 


\section{AUTHOR NOTE}

Parts of this article were presented at the European Conference on Perception in Trieste (1999). The abstract was published in the supplement of Perception (Vol. 28). We thank Michael J. Morgan and two anonymous reviewers for valuable comments on an earlier draft of the manuscript. This work was supported in part by Grant DFG La 564/10-4 from the Deutsche Forschungsgemeinschaft to H.L. Correspondence concerning this article should be addressed to I. Ludwig, Faculty of Psychology, Philipps-Universität Marburg, Gutenbergstrasse 18, D-35032 Marburg, Germany (e-mail: ludwigi@staff.uni-marburg.de).

\section{REFERENCES}

Anstis, S. M. (2000). Monocular lustre from flicker. Vision Research, 40, 2551-2556.

AULHORN, E. (1971). Psychophysische Grundlagen des Binokularsehens [Psychophysical principles of binocular vision]. In O.-J. Grüsser \& R. Klinke (Eds.), Zeichenerkennung durch biologische und technische Systeme: Tagungsbericht des 4. Kongresses der Deutschen Gesellschaft für Kybernetik durchgeführt an der Technischen Universität Berlin vom 6.-9. April 1970 (pp. 262-275). Berlin: Springer.

Barlow, H. B., Blakemore, C., \& Pettigrew, J. D. (1967). The neural mechanism of binocular depth discrimination. Journal of Physiology, 193, 327-342.

BuRR, D. C., \& Ross, J. (1979). How does binocular delay give information about depth? Vision Research, 19, 523-532.

DVORÁK, V. vON (1872). Uber Analoga der persönlichen Differenz zwischen beiden Augen und den Netzhautstellen desselben Auges [On analogues of the personal difference between both eyes and the retinal points of the same eye]. Sitzungsberichte der Königlichen Böhmischen Gesellschaft der Wissenschaften, 65-74.

EFron, R. (1957). Stereoscopic vision: I. Effects of binocular temporal summation. British Journal of Ophthalmology, 41, 709-730.

ENGEL, G. R. (1970). An investigation of visual responses to brief stereoscopic stimuli. Quarterly Journal of Experimental Psychology, 22, 148-166.

EWALD, J. R., \& Gross, O. (1906). Über Stereoskopie und Pseudoskopie [On stereoscopic vision and pseudoscopic vision]. Pflügers Archiv für die gesamte Physiologie, 115, 514-532.

EXNER, S. (1875). Experimentelle Untersuchung der einfachsten psychischen Processe [Experimental investigation of the simplest psychological processes]. Pflügers Archiv für die gesamte Physiologie, 11, 403-432.

Fendick, M., \& Westheimer, G. (1983). Effects of practice and the separation of test targets on foveal and peripheral stereoacuity. Vision Research, 23, 145-150.

GuILLOZ, T. (1904). Sur la stéréoscopie obtenue par les visions consécutives d'images monoculaires [On stereopsis obtained by perception of successive monocular images]. Comptes rendus des séances de la Société de Biologie et de ses filiales, 56, 1053-1054.

Helmholtz, H. von (1910). Handbuch der physiologischen Optik [Handbook of physiological optics] (3rd ed., Vol. 3). Leipzig: Voss.

Herzau, V. (1976). Stereosehen bei alternierender Bilddarbietung [Stereo vision with alternating presentation of images]. Albrecht von Graefes Archiv für Ophthalmologie, 200, 85-91.

Howard, I. P. (1995). Depth from binocular rivalry without spatial disparity. Perception, 24, 67-74.

Howard, I. P., \& Rogers, B. J. (1995). Binocular vision. Oxford: Oxford University Press, Clarendon Press.

Julesz, B. (1960). Binocular depth perception of computer-generated patterns. Bell System Technical Journal, 39, 1125-1162.

Julesz, B. (1971). Foundations of cyclopean perception. Chicago: University of Chicago Press.
Julesz, B., \& White, B. (1969). Short term visual memory and the Pulfrich phenomenon. Nature, 222, 639-641.

LEVELT, W. J. M. (1965). Binocular brightness averaging and contour information. British Journal of Psychology, 56, 1-13.

MсCАмY, C. S. (1998). Observation and measurement of the appearance of metallic materials: Part II. Micro appearance. Color Research \& Application, 23, 362-373.

Milgram, P. (1987). A spectacle-mounted liquid-crystal tachistoscope. Behavior Research Methods, Instruments, \& Computers, 19, $449-456$.

Mollon, J. D., Polden, P. G., \& Morgan, M. J. (1977). Electro-optic shutters and filters. Quarterly Journal of Experimental Psychology, 29, 147-156.

Morgan, M. J. (1979). Perception of continuity in stroboscopic motion: A temporal frequency analysis. Vision Research, 19, 491-500.

Morgan, M. J. (1981). Vernier acuity and stereopsis with discontinuously moving stimuli. Acta Psychologica, 48, 57-67.

Morgan, M. J., \& CASTET, E. (1995). Stereoscopic depth perception at high velocities. Nature, 378, 380-383.

Morgan, M. J., \& FAHLE, M. (2000). Motion-stereo mechanisms sensitive to inter-ocular phase. Vision Research, 40, 1667-1675.

MorGan, M. J., \& WARD, R. (1980). Interocular delay produces depth in subjectively moving noise patterns. Quarterly Journal of Experimental Psychology, 32, 387-395.

OGLE, K. N. (1963). Stereoscopic depth perception and exposure delay between images to the two eyes. Journal of the Optical Society of America, 53, 1296-1304.

OGLE, K. N., \& ReIHER, L. (1962). Stereoscopic depth perception from after-images. Vision Research, 2, 439-447.

O'SheA, R. P., \& CRASSini, B. (1984). Binocular rivalry occurs without simultaneous presentation of rival stimuli. Perception \& Psychophysics, 36, 266-276.

PetTigrew, J. D., Nikara, T., \& Bishop, P. O. (1968). Binocular interaction on single units in cat striate cortex: Simultaneous stimulation by single moving slit with receptive fields in correspondence. Experimental Brain Research, 6, 391-410.

PIEPER, W., \& LUDWIG, I. (1998). Monocular stimulation conditions and stereoacuity. Perception, 27(Suppl.), 104.

PiePer, W., \& LudWig, I. (2001). Binocular vision: Rivalry, stereoscopic lustre, and sieve effect. Perception, 30(Suppl.), 75-76.

Pulfrich, C. (1922). Die Stereoskopie im Dienste der isochromen und heterochromen Photometrie [Stereopsis in the service of isochromic and heterochromic photometry]. Naturwissenschaften, 10, 553-564.

Qian, N., \& ANDERSON, R. A. (1997). A physiological model for motion-stereo integration and a unified explanation of Pulfrich-like phenomena. Vision Research, 37, 1683-1698.

Ross, J., \& HoGBEN, J. H. (1974). Short-term memory in stereopsis. Vision Research, 14, 1195-1201.

STEVENSON, T. J., \& SANFORD, E. C. (1908). A preliminary report of experiments on time relations in binocular vision. American Journal of Psychology, 19, 130-137.

Wallach, H., Moore, M. E., \& Davidson, L. (1963). Modification of stereoscopic depth-perception. American Journal of Psychology, 76, 191-204.

WESTHEIMER, G., \& McKeE, S. P. (1979). What prior uniocular processing is necessary for stereopsis? Investigative Ophthalmology \& Visual Science, 18, 614-621.

WIST, E. R., \& GoGEL, W. C. (1966). The effect of interocular delay and repetition interval on depth perception. Vision Research, 6, 325-334.

WoOdWORTH, R. S., \& SchlosberG, H. (1964). Experimental psychology. New York: Holt, Rinehart \& Winston.

(Manuscript received January 24, 2005; revision accepted for publication March 7, 2006.) 